\title{
Which Techniques Should Be Used to Screen Blood Donations For Hepatitis B Surface Antigen?
}

\author{
J. WALLACE, A. BARR, G. R. MILNE
}

\section{Donors and Methods}

Over four years 438937 donations were screened for HBsAg by C.I.E.P. ${ }^{3} 165811$ of them for the first time. Any donor confirmed to be positive for HBsAg is advised not to donate again so 273126 donations were from people who had already been found to be negative for HBsAg.

All donations found to be negative for HBsAg by C.I.E.P. were available for issue to hospitals, but some serum from each donor was preserved frozen for one year for subsequent investigation. Later 27487 of these preserved sera were tested for HBsAg by R.P.H.A. (16 006 sera by Hepanosticon, ${ }^{4}$ Organon Laboratories; 5050 by Auscell, ${ }^{5}$ Abbott Laboratories; and 6431 by Hepatest, ${ }^{6}$ Wellcome). Of these 27487 sera 22239 were also tested by R.I.A. (16 006 by Ausria $\mathrm{I}^{7}{ }^{7}$ Abbott Laboratories, and 6233 by Ausria $\mathrm{II}^{8}$ ). Incubation at $45^{\circ} \mathrm{C}$ instead of room temperature was used with the R.I.A. reagents. ${ }^{9}$

Confirmatory tests, including specific neutralization of suspected HBsAg by potent anti-HBs, ${ }^{9}$ on sera which gave positive reactions in any of the screening tests were performed according to the techniques recommended in earlier publications on each method. Sera giving presumptive positive reactions on any one technique were retested by all three.

We tried to discover the fate of any donation which was negative for HBsAg by C.I.E.P. but subsequently found to be positive by R.P.H.A. or R.I.A. The recipients of such donations were investigated for manifestations of hepatitis including $\mathrm{HBsAg}$ or anti-HBs in the serum. positive reactions when using R.P.H.A. or R.I.A. for screening was less than $1 \%$. As many as 700 donations in one day have been tested by R.P.H.A. or R.I.A. R.P.H.A. is faster and less expensive than R.I.A., but R.I.A. is more objective. Either R.P.H.A. or R.I.A. should replace C.I.E.P. as the routine method of screening donor sera for HBsAg.

\section{Introduction}

The transmission of viral hepatitis such as hepatitis type $\mathrm{B}^{\mathbf{1}}$ is the most serious complication of the use of blood products. The Australia or hepatitis $B$ surface antigen $(\mathrm{HBsAg})$ is a specific marker of infection with the agent of viral hepatitis $B$, and donors with HBs antigenaemia should be excluded. A World Health Organization report has called for a simple, rapid, sensitive, and specific method for detecting $\mathrm{HBsAg}$ in blood donors, ${ }^{2}$ and recommended as a desired minimum a method with the sensitivity of counterimmunoelectrophoresis (C.I.E.P.). A screening test's sensitivity, its standard of performance, and the specificity of the reagents obviously influence the rate of detection of HBsAg in donors, but the W.H.O. report maintains that most apparently healthy carriers have a high titre of $\mathrm{HBsAg}$ which is easily detected by relatively insensitive methods such as C.I.E.P.

We reviewed our four years' experience of screening all donations for HBsAg with C.I.E.P. and compared C.I.E.P. with two other screening tests-reverse passive haemagglutination (R.P.H.A.) and radioimmunoassay (R.I.A.).

Glasgow and West of Scotland Blood Transfusion Centre, Law Hospital, Carluke, Lanarkshire ML8 5ES

J. WALLACE, M.D., F.R.C.P., Regional Director

A. BARR, F.I.M.L.T., Chief Technician

G. R. MILNE, F.P.S., Honorary Consultant

We screened another 22200 donations for HBsAg by all three techniques; C.I.E.P. detected 25 positive donors, R.P.H.A. 35, and R.I.A. 37. Another 5287 donations were screened by C.I.E.P. and R.P.H.A., which detected, respectively, two and four positive samples. Of these 5287 donations $39-$ those which gave presumptive positive reactions on R.P.H.A.-were also tested with R.I.A., which con- 
firmed as positive only the four already confirmed by R.P.H.A Thus of the 22239 donations tested by all three methods C.I.E.P. detected 27 positive for HBsAg, R.P.H.A. detected 39, and R.I.A. detected 41 . There were no false positives on C.I.E.P., $181(0.66 \%)$ on R.P.H.A., and $91(0.41 \%)$ on R.I.A.

Thus 14 donations which had been available for normal transfusion on the strength of a negative reaction on C.I.E.P. were found to be HBsAg positive by R.I.A. Six of the donations were not used or not traced, and two were given to patients who died soon after transfusion. One donation had been processed to provide cryoglobulinprecipitate (factor VIII), parts of which were given to two recipients; one developed anicteric and the other icteric hepatitis. The evidence suggested that viral hepatitis type B was transmitted to four other recipients, one of whom developed an anicteric illness. One of the recipients with anicteric hepatitis became a chronic carrier of $\mathrm{HBsAg}$ and one of those with icteric hepatitis died of acute hepatic necrosis. The recipient of the remaining donation remained well and seronegative.

\section{Discussion}

The three main reasons for testing all donations for HBsAg are (a) to prevent serious and sometimes fatal hepatitis in recipients; (b) to protect medical, nursing, and technical staff and patients' relatives from the infective agent; and $(c)$ in the interests of public health; the transfusion of blood containing $\mathrm{HBsAg}$ may produce an asymptomatic illness and a persistent antigenaemia and thus increase the circulation of the infective agent in the population.

The routine C.I.E.P. method, ${ }^{3}$ which is simple, fast, and cheap, detected $217 \mathrm{HBsAg}$-positive donors in the west of Scotland over four years, and only nine seemed to be on the fringe of detectability by C.I.E.P., four being of high titre and five of low titre. Nevertheless, the nine examples of $\mathrm{HBsAg}$ on the fringe of detectability by C.I.E.P. caused nine falsenegative reactions on the original screening, which resulted in at least four cases of post-transfusion hepatitis B. The sera giving these nine false-negative reactions by C.I.E.P. contained HBsAg which was readily detectable by all the R.P.H.A. and R.I.A. methods we used.

\section{MORE SENSITIVE SCREENING}

Preliminary evidence has suggested that the effects of introducing more sensitive screening techniques for $\mathrm{HBsAg}$ would not be great, ${ }^{2}$ and Arndt-Hanser et al. ${ }^{10}$ consider that compulsory screening should not be performed unless there is strong evidence that the number of units of infectious blood can be reduced. Prince et al. ${ }^{11}$ found that in 204 cardiovascular patients an agent other than $\mathrm{HBsAg}$ had caused 36 out of 51 cases of posttransfusion hepatitis. But as well as the 15 patients with hepatitis B infection another 25 had serological evidence of exposure to HBsAg without developing hepatitis.

The Public Health Laboratory Service supports the introduction of more sensitive methods of testing blood for HBsAg to prevent transmission of infection by transfusion, ${ }^{12}$ and our results have confirmed that both R.P.H.A. and R.I.A. are more sensitive techniques than C.I.E.P. ${ }^{7}$ 13-15 At least six recipients of our C.I.E.P.-negative but R.P.H.A.and R.I.A.-positive blood developed hepatitis, and one died of acute liver failure. Another recipient became a chronic carrier of $\mathrm{HBsAg}$, thus exposing contacts in hospital and at home to infection. One donation caused hepatitis in two recipients, and with the development of component therapy more and more donations will be given to several recipients, ${ }^{16}$ and thus aggravate the danger of false negative donations. Hence, R.P.H.A. or R.I.A. should replace C.I.E.P. for screening blood donations for HBsAg.

\section{WHICH METHOD?}

When ultrasensitive screening methods are used specific confirmatory tests for HBsAg should be performed, " as the label "chronic hepatitis-virus carrier" may be professionally and socially injurious. Neutralization of $\mathrm{HBsAg}$-containing sera with potent anti-HBs is an essential check on specificity. We found that $0.66 \%$ of sera were falsely positive on R.P.H.A. and $0.41 \%$ on R.I.A., and most of the false-positive reactions on R.I.A. occurred with Ausria I. With Ausria II false-positive reactions are infrequent. These levels of false-positive reactions are acceptable as $99 \%$ of the donations are available for issue to hospitals on the day of testing.

The largest number of donations tested by C.I.E.P. was 650 in one morning, the usual range being from 350 to $450 .^{8}$ Similar numbers could be tested by each of the three R.P.H.A. methods used and by R.I.A. Vandervelde et al. ${ }^{17}$ claim that R.P.H.A. is a suitable and convenient technique for routine screening of donations by a transfusion centre but consider that R.I.A. is laborious though valuable for confirmatory testing. R.P.H.A. is as rapid as C.I.E.P. and the small number of essential R.P.H.A. confirmatory tests, including specific neutralization by anti-HBs, can be completed the same day as the screening tests or the next morning. R.I.A. testing is admittedly slower than C.I.E.P. or R.P.H.A. but not laborious. As many as 700 donations have been tested by two skilled technicians in one day. Incubation at $45^{\circ} \mathrm{C}$ reduced the standard 18-hour room temperature incubation to two hours without loss of sensitivity and fewer false-positive reactions. Thus by 5 p.m. on the day of screening by the rapid R.I.A. test at least 150 donations of fresh whole blood were available for issue to hospitals, and these were enough to meet demands. By using an automatic gamma counter the remaining tests were completed overnight. Technical staff have not found R.I.A. laborious, and they appreciate the objective reading of the results of R.I.A. compared with the subjectivity of C.I.E.P. and R.P.H.A.

Our results suggest that R.I.A. is slightly more sensitive than R.P.H.A. The number of false-positive reactions with Austria II is less than with any of the R.P.H.A. methods, but even with R.P.H.A. the number of false-positive reactions is acceptable when screening blood donations, and R.I.A. is slower than R.P.H.A. The main disadvantage of R.I.A. is the cost not only of reagents but also of equipment. To make R.I.A. more acceptable to transfusion services by reducing the time taken to complete screening tests, two automatic gamma counters would be needed, which would also be useful in case of breakdowns. Transfusion centres must complete the screening tests on all donations in one day because a similar large volume of work has to be undertaken the next and each following day. An important practical point is the need to use serum rather than plasma for R.P.H.A. and R.I.A. False positives are often obtained when citrated plasma specimens are used. The difficulty can be overcome by recalcifying the plasma samples, but recalcification is impracticable and time consuming for large numbers. It is therefore essential with R.P.H.A. and R.I.A. to test serum samples.

Since R.P.H.A. is simple and does not need expensive equipment it can be introduced quickly and more cheaply than R.I.A. Thus, we recommend that R.P.H.A. should replace C.I.E.P. as the method of screening donations for $\mathrm{HBsAg}$ and that transfusion centres should have easy access to a reference laboratory with facilities for R.I.A. testing of specimens giving equivocal results on R.P.H.A.

We thank colleagues in Abbott Laboratories, Organon Laboratories, and the Wellcome Foundation for the opportunity of evaluating their diagnostic reagents and for their many helpful discussions. We also thank colleagues in west of Scotland hospitals who helped us to trace recipients. The work was supported by a special grant from the Scottish National Blood Transfusion Association.

\section{References}

1 Maycock, W. d'A., British Medical Bulletin, 1972, 28, 163.

2 World Health Organization, Viral Hepatitis, Technical Report Series, No. 512, Geneva, W.H.O., 1973. 
3 Wallace, J., Milne, G. R., and Barr, A., British Medical fournal, 1972, $1,663$.

4 Schuurs, A. H. W. M., and Kacaki, J., Vox Sanguinis, 1974, 27, 97.

5 Jarboe, R. H., personal communication.

6 Cayzer, I., et al., Lancet, 1974, 1, 947.

7 Prince, A. M., et al., Lancet, 1973, 1, 1346

8 Prince, A. M., et al., Lancet, 1973, 1,

9 Prince, A. M., and Jass, D., Vox Sanuinis, 1974, 26, 209.

10 Arndt-Hanser, A., et al., Lancet, 1974, 2, 345.
11 Prince, A. M., et al., Lancet, 1974, 2, 241.

12 Public Health Laboratory Service, British Medical fournal, 1974, 4, 751.

13 Ling, C. M., and Overby, L. R., Fournal of Immunology, 1974, 109, 834

14 Reesink, H.' W., Duimel, W. J., and Brummelhuis, H. G. J., Lancet, 1973, 2, 1351.

15 Wallace, J., Lancet, 1973, 1, 1002.

16 Wallace, J., Proceedings of the Royal Society of Edinburgh, 1972, 71, s7.

17 Vandervelde, E. M., et al., Lancet, 1974, 2, 1006.

\title{
Plasma Nicotine Levels after Smoking Cigarettes with High, Medium, and Low Nicotine Yields
}

\author{
M. A. H. RUSSELL, \\ C. WILSON, \\ U. A. PATEL, \\ C. FEYERABEND, P.V. COLE
}

British Medical fournal, 1975, 2, 414-416

\begin{abstract}
Summary
Plasma nicotine three minutes after smoking a cigarette was measured in 10 sedentary workers in mid-morning and five hours later on four typical working days. The average mid-morning level after they had been smoking their usual cigarettes (mean nicotine yield $1.34 \mathrm{mg}$ ) was $150.4 \mathrm{nmol} / 1$ (24.4 $\mathrm{ng} / \mathrm{ml}$ ) (range 95.6-236.7 nmol/1 (15.5$38.4 \mathrm{ng} / \mathrm{ml}$ )). Despite great variation between smokers the mid-morning levels of each smoker were fairly consistent over the four mornings and correlated 0.82 with their carboxyhaemoglobin levels. After continuing to smoke their usual brand or switching to a high-nicotine brand (3.2 $\mathrm{mg}$ ) average afternoon levels of 185.6 and $180.0 \mathrm{nmol} / 1(30.1$ and $29.2 \mathrm{ng} / \mathrm{ml})$ respectively were not significantly higher than the morning levels, but after switching to low-nicotine cigarettes $(0 \cdot 14 \mathrm{mg})$ the plasma nicotine dropped to an average of $52.4 \mathrm{nmol} / 1(8.5 \mathrm{ng} / \mathrm{ml})$. The changes between morning and afternoon while smoking usual or high-nicotine cigarettes showed marked individual variation. The findings suggest that the plasma nicotine level just after a cigarette depends more on the way the cigarette is smoked than on its nicotine yield or the number which have been smoked over the preceding few hours.
\end{abstract}

\section{Introduction}

Official tables listing the tar and nicotine yields of cigarettes are based on the rigidly standardized and unvaried puffing of smoking machines. This is entirely appropriate for the comparison of cigarette brands but has trapped many into assuming too directly the implications for less hazardous smoking. Almost universally the public are recommended that, in the interest of their health, if they cannot stop smoking cigarettes they should try to switch to the low-tar, low-nicotine brands.

But people are not smoking machines. Even though some smokers may take a $35-\mathrm{ml}$, two-second puff once a minute to a butt length of $20 \mathrm{~mm}$ or the tip overwrap plus $3 \mathrm{~mm}$, which-

\footnotetext{
Addiction Research Unit, Institute of Psychiatry, Maudsley Hospital, London SE5 8AF

M. A. H. RUSSELL, M.R.C.P., M.R.C.PSYCH., Senior Lecturer and Honorary

Consultant
C. WILSON, B.SC., Research Worker

C. WILSON, B.SC., Research Wo
U. A. PATEL, M.SC., Statistician

Poisons Unit, New Cross Hospital, London S.E.14

Poisons Unit, New Cross Hospital, Lond

St. Bartholomew's Hospital, London, E.C.

P. V. COLE, M.B., F.F.A. R.C.S., Consultant Anaesthetist
}

ever is the greater, there is no guarantee that they will smoke a cigarette in the same way irrespective of its nicotine yield. Indeed, the evidence is to the contrary. Smokers terd to respond to changes in the tar and nicotine yields of cigarettes by altering their smoking pattern to regulate the nicotine intake. ${ }^{1-3}$ When the nicotine yield is high they tend to smoke less, and when it is low they compensate by smoking more. Since tar and nicotine yields of currently available cigarettes correlate so highly $(r=0.96)$ it has still to be proved that the adjustment relates to nicotine and not to tar or some other covarying component.

Apart from the number of cigarettes smoked there are more subtle ways in which a smoker may consciously or unconsciously alter his nicotine intake. Puff-size, puff-velocity, puff-rate, number of puffs per cigarette, proximity of puffs to tip, proportion of puff inhaled, and depth and duration of inhalation are some of the more obvious factors. Hitherto, the most sophisticated measure of nicotine intake has been by the analysis of nicotine in cigarette butts, ${ }^{34}$ which makes use of the close linear relation between the amount of nicotine deposited in the filter and the amount which passes through it. But even this tells us only how much the smoker has taken into his mouth.

For quantitative study the measurement of nicotine in urine is limited by the $\mathrm{pH}$ dependence of its excretion, and for various reasons it has been difficult to measure in blood. A reliable blood method has now been developed, ${ }^{5}$ and we have investigated the plasma nicotine levels in different smokers and the degree to which an individual smoker shows a consistent level on different days, at different times of the day, and when smoking cigarettes with different nicotine yields.

\section{Subjects and Methods}

Ten clerical and social workers volunteered to take part in the study. They were all regular cigarette smokers who said that they inhaled deeply. Venous blood samples were taken between 10 a.m. and $11.30 \mathrm{a} . \mathrm{m}$. and again about five hours later ( 3 p.m. to 4.30 p.m.) on four typical working days: two days of one week (days 1 and 2) and the same two days of the following week (days 3 and 4). For each subject the blood was taken at the same time on all four days. To avoid variations due to different time-spans between the last cigarette and collection of the samples the volunteers smoked a cigarette immediately before each sample, which was then taken three minutes after completion of the cigarette. On the morning of each day of study the subjects smoked their usual brand in the usual way. For the five hours between sampling they were given a liberal supply of cigarettes with instructions to smoke as much or as little as they wanted but not to offer or accept cigarettes from other people. On days 1 and 3 they were given their usual brand, but on days 2 and 4 they were given a high- or low-nicotine cigarette according to a balanced crossover design.

The high nicotine cigarette used was Capstan Full Strength (tar $38 \mathrm{mg}$, nicotine $3.2 \mathrm{mg}$ ) and the low nicotine cigarette Silk Cut 